\title{
Rational Disinhibition and Externalities in Prevention
}

\author{
Flavio Toxvaerd*
}

January 30, 2019

\begin{abstract}
This paper studies a model of disease propagation in which individuals can control their exposure to infection by engaging in costly preventive behavior. Individuals are fully rational, strategically sophisticated and forwardlooking. Equilibrium outcomes under decentralized decision making are characterized and contrasted to the outcomes chosen by a benevolent social planner. In general, individuals over-expose themselves to infection, leading to suboptimally high disease prevalence. The model is applied to study the welfare effects of preexposure prophylaxis, which reduces transmission between serodiscordant couples and causes disinhibition. It is shown that a decrease in the induced infection risks increases disease prevalence and can lead to decreases in overall welfare.

JEL Classification: C73, I18.

KEYWORDS: Economic epidemiology, preventive behavior, disinhibition, immiserization, second-best policies.
\end{abstract}

\section{INTRODUCTION}

Historically, infectious diseases have posed a major obstacle to human wellbeing, causing both morbidity and mortality to human populations. Despite significant progress in understanding diseases and developing medicines to combat their spread, epidemic outbreaks continue to be an issue of first order importance for public policy. The recent introduction of pre-exposure prophylaxis, such as the drug Truvada, has been lauded as a "game changer". Such drugs decrease the probability that infection is transmitted during an unprotected encounter and have raised expectations that it may radically alter the calculus of HIV control. ${ }^{1}$

Regrettably, there is a considerable lack of clarity about both the likely effects and the desirability of such medical innovations and the issue of so-called risk compensation remains controversial. Risk compensation, or disinhibition, is said to occur if a decision maker responds to decreased risk by engaging in more risky behavior.

In this paper, I consider second-best interventions such as pre-exposure prophylaxis and their relation to disinhibition in a susceptible-infected-susceptible (SIS) type model. My analysis contributes to this important policy debate by addressing the following two fundamental questions: Does the introduction of pre-exposure prophylaxis to the uninfected (i) cause disinhibition? and/or (ii) decrease social welfare?

To preview the answer to the first question, my analysis confirms that a permanent decrease in the infectiousness of the disease will prompt an increase in exposure and in steady state disease prevalence. The reason is that such a change alters the tradeoffs faced by decision makers in favour of increased exposure. Although the decrease in infectiousness decreases the rate of transmission per exposure, the exposure itself increases so

\footnotetext{
${ }^{*}$ Faculty of Economics, University of Cambridge. Email: fmot2@cam.ac.uk.

${ }^{1}$ See e.g. The Guardian, August 2, 2016: Judge to rule on NHS funding for 'game-changing' HIV treatment and BBC, February 25, 2015: Analysis: An HIV 'game changer'?
} 
much that the net effect is to increase disease incidence. This outcome is not pathological and holds both for a utilitarian social planner and for self-interested individuals.

To preview the answer to the second question, my analysis shows that a decrease in the infectiousness of the disease may indeed lead to a decrease in social welfare under certain conditions. The reason is that individuals make different choices than those preferred by a social planner. There are two sources for this discrepancy. First, there is a pure externality effect that arises because individuals do not internalize the benefits to others that flow from the individual protecting itself. As a consequence, aggregate equilibrium protection is too low, thus causing higher future disease prevalence. This means that the equilibrium future path of infection faced by the individual is higher than the path preferred (and indeed chosen) by a social planner. But since each individual takes the path of aggregate infection as given, this means that the individual and the planner face different future paths of infection and this causes a second discrepancy between the optimal choices and the equilibrium choices. This further drives a wedge between optimal and equilibrium infection paths. This second effect stems from the fact that each (small) individual takes aggregate disease prevalence as given.

These effects mean that the introduction of pre-exposure prophylaxis may well lead to a decrease in social welfare. That this possibility can materialize is verified numerically.

These findings point to two central policy conclusions. First, the customary focus on disinhibition in the policy debate is misplaced. When one decreases the infectiousness of a disease, it may be perfectly desirable that individuals increase exposure and hence cause disease prevalence to rise. After all, to the extent that individuals derive utility from such exposure, it is right that policy should take that into account when trading off the costs and benefits of such exposure. Second, the presence of external effects may lead policy interventions to have undesirable consequences. In particular, a well-intentioned secondbest policy to reduce infections may in fact exacerbate the problem of over-exposure and lead to socially inferior outcomes.

The present work contributes to a growing literature that studies the effects and desirability of permanently decreasing the infectiousness of diseases through medical innovations. Lakdawalla et al. (2006) consider the effects of simultaneously introducing antiretroviral therapy to susceptible and infected individuals in a susceptible-infected (SI) type model. They note that there are several confounding effects. Ceteris paribus, the susceptible individuals may benefit, because the introduction of antiretrovirals effectively reduces the probability of transmission per risky sexual act. On the other hand, antiretrovirals increase the survival probability of infected individuals, thereby increasing the source of infection. Unfortunately, Lakdawalla et al. (2006) do not distinguish between equilibrium outcomes and socially optimal outcomes and it is therefore not a priori clear from their work that policy interventions are warranted in the first place. ${ }^{2}$ Chan et al. (2018) consider the effects of introducing antiretroviral therapy to susceptible individuals and conclude that they are likely to benefit from reduced infectivity. Gersovitz (2010) considers innovation in an infection context, but mainly offers results for technological improvements that do not interact directly with individual's decisions. The technological improvements he considers cannot be interpreted as pre-exposure prophylaxis and his analysis is therefore not directly comparable to that in this paper. Last, Tamalas and

\footnotetext{
${ }^{2} \mathrm{~A}$ consequence of the analysis in the present paper is that when no recovery is possible, i.e. in a standard SI model, equilibrium is socially optimal unless the population is heterogeneous.
} 
Vohra (2018) consider the effects if imperfect protection in a network-theoretic model of partner choice and show that a decrease in infectiousness can cause welfare-reducing changes in partnership formation.

Disinhibition and related offsetting effects have been noted in several different contexts. In energy and conservation economics, the so-called rebound effect has been recognized as a real possibility (see Gillingham et al., 2015 for a review of this literature). The rebound effect is said to be present if an innovation that is intended to reduce the use of a resource instead acts to increase the use of said resource. The rebound effect is also known as the Peltzman effect, articulated in the context of safety equipment and driving (see Peltzman, 1975 and Hoy and Polborn, 2015 for a more general and abstract treatment). In traffic and network economics, the "fundamental law of road congestion" posits that an improvement of a road network may lead to increased congestion (Duranton and Turner, 2011). In the context of protection against infectious diseases, such effects are known as disinhibition. Interestingly, an almost universal feature of papers on disinhibition focus on whether the effect is present and do not deal with the welfare consequences of such effects (see e.g. Kremer, 1996, Richens et al., 2000 and Blumenthal and Haubrich, 2014). Notable exceptions are Chan and Gillingham (2015), who analyze the welfare consequences of the rebound effect in a static model of energy use and Hoy and Polborn (2015), who analyze the effects of innovations and externalities in an abstract, static two-player game. ${ }^{3}$

The formal framework of my analysis is that of an economic epidemiology model with disease prevention. ${ }^{4}$ There is a relatively large and growing literature on the effects and desirability of preventive measures such as quarantines, prophylaxis, vaccines and promiscuity/abstinence. Main contributions include Sethi (1978), Geoffard and Philipson (1996), Kremer (1996), Auld (2003), Gersovitz and Hammer (2004), Aadland et al. (2013), Francis (2004), Reluga (2009, 2010). Chen and Toxvaerd (2014) surveys the large literature on the economics of vaccination. Although many of these papers study interventions that fall under the broad category of "preventive measures" that may seem superficially similar, there are important differences between them in terms of predictions and policy recommendations.

Last, this paper departs from most of the existing literature in another respect, namely in its comprehensive analysis of the dynamics of the disease. It is difficult to analyze fully dynamic models of disease propagation analytically and it is therefore common to focus the analysis on the steady state(s) of such systems. Papers that take this route include Kremer (1996), Barrett (2003), Kremer and Snyder (2003), Auld (2003), Lakdawalla et al. (2006), Greenwood et al. (2013), Cerdeiro (2017) and Bate et al. (2016). No doubt, the steady state(s) of a dynamic system are important, but to focus exclusively on steady states can lead to misleading policy conclusions.

In Section 2, I set out the classical and economic models and summarize the classical results. In Section 3, I analyze the problem of a benevolent central planner. In Section 4, I analyze the equilibrium under non-cooperative decentralized decision making. In Section 5, I discuss welfare, derive comparative statics results and draw policy conclusions. In

\footnotetext{
${ }^{3}$ Duranton and Turner (2009) perform a related static welfare analysis in the context of road congestion.

${ }^{4}$ For a review of the related but distinct literature on the effects of treatment, see Rowthorn and Toxvaerd (2015).
} 
Section 6, I discuss the extension to settings with a heterogeneous population. In Section 7, I discuss the main policy recommendations and offer concluding remarks. Appendix A contains some proofs and an additional online Appendix contains details of omitted proofs and of illustrative numerical experiments.

\section{The Classical and Economic Models}

The classical susceptible-infected-susceptible model is simple to describe. Time is continuous and the horizon is infinite. A population $\mathcal{P}=[0,1]$ consists of a continuum of infinitely lived individuals who can at each instant $t \geq 0$ each be in one of two states, namely susceptible or infected. The set of infected individuals is denoted by $\mathcal{I}(t)$ and has measure $I(t)$, while the set of susceptible individuals is denoted by $\mathcal{S}(t)$ and has measure $S(t)$. Because the population size is normalized to unity, these measures can be interpreted as fractions. In what follows, $I(t)$ will be referred to as disease prevalence.

At each instant, the population mixes homogeneously. This corresponds to random matching, where each individual has an equal chance of meeting any other individual, irrespective of the health status of the two matched individuals. A match between two infected individuals or two susceptible individuals does not create any new infection, but a match between an infected and a susceptible individual may. The rate at which infection is transferred in such a match is denoted by $\beta>0$. This parameter captures the infectivity of the disease. Coupled with the assumption of homogeneous mixing, this means that the aggregate rate at which susceptible individuals become infected is $\beta I(t) S(t)$. Thus the rate of new infection, or disease incidence, is proportional to disease prevalence. ${ }^{5}$

Infected individuals recover spontaneously at rate $\alpha \geq 0$. Thus the aggregate rate at which infected individuals become susceptible is $\alpha I(t)$. The dynamics of the model are described by the system of differential equations

$$
\begin{aligned}
\dot{S}(t) & =I(t)[\alpha-\beta S(t)] \\
\dot{I}(t) & =I(t)[\beta S(t)-\alpha] \\
I(t) & =1-S(t), \quad I(0)=I_{0}
\end{aligned}
$$

Since the size of the population is normalized to one, this system reduces to the following logistic growth equation:

$$
\dot{I}(t)=I(t)[\beta(1-I(t))-\alpha], \quad I(0)=I_{0}
$$

The steady states of this system are given by

$$
I^{*}=0, \quad I^{*}=\frac{\beta-\alpha}{\beta}
$$

For $\beta>\alpha$, the stable steady state is endemic (or persistent), while for $\beta<\alpha$, the stable steady state involves eradication. In the special case $\alpha=0$, the unique steady state has

\footnotetext{
${ }^{5}$ The term $\beta I(t) S(t)$ should be thought of as the rate at which susceptible individuals have contact with other individuals, multiplied by the probability of the contact being with an infectious individual, multiplied by the probability that the infection is transmitted in such a contact. See e.g. Keeling and Rohani (2008) for a detailed derivation.
} 
everyone infected.

At the aggregate level, there is no uncertainty and thus the probability that an arbitrary individual is infected coincides with the fraction of infected individuals. From the perspective of an infected individual, the transition to susceptibility is governed by a Poisson process with rate $\alpha$. Similarly, for a fixed level of aggregate infection $I(t)$, the transition to infectivity for a susceptible individual is governed by a Poisson process with rate $\beta I(t)$. Thus transition probabilities are memoryless, a fact that greatly simplifies the analysis that follows.

Note that in the classical model, the endemic steady state level of disease prevalence is decreasing in the recovery rate $\alpha$ and increasing the infectivity rate $\beta$. While these comparative statics are intuitively appealing, it turns out that they are misleading as a guide for conducting policy.

2.1. Extension to an Economic Model. To turn the classical model into an economic model, I will assume that individuals earn a flow payoff $\pi_{\mathcal{S}}>0$ per instant while susceptible, a flow payoff $\pi_{\mathcal{I}}<\pi_{\mathcal{S}}$ per instant while infected and that time is discounted at rate $\rho>0$. For notational simplicity, let $\pi \equiv \pi_{\mathcal{S}}-\pi_{\mathcal{I}}>0$ denote the health premium. The health premium should be thought of broadly as the benefits of not being infected, e.g. physical well-being, higher productivity etc.

To model the possibility of engaging in preventive behavior, assume that the individuals can affect the rate of infection by controlling the rate at which they expose themselves to infection. In particular, at each instant $t \geq 0$, each susceptible individual $i \in \mathcal{S}(t)$ non-cooperatively chooses exposure level $\varepsilon_{i}(t) \in[0,1]$ at personal cost $\left(1-\varepsilon_{i}(t)\right) c \geq 0 .{ }^{6}$ Effectively, this reduces the rate of infection to $\varepsilon_{i}(t) \beta I(t)$. This formalization captures the notion that, ceteris paribus, exposure is desirable. Equivalently, this means that engaging in preventive behavior is privately costly. In the absence of infection risk, it would be optimal to choose full exposure and to not protect oneself.

The assumption of linear costs will greatly simplify the analysis. The main results of the analysis remain qualitatively unchanged under more general convex cost structures, but at the expense of considerable analytical complexity and at the loss of closed-form solutions for steady state values of control and state variables. Throughout, I will state which main results rely on the linearity assumption.

To make the problem interesting, the following assumptions are maintained throughout:

\section{Assumption 1}

$$
c<\frac{\beta \pi}{\alpha+\beta+\rho}
$$

This assumption imposes an upper bound on the cost of prevention that ensures that prevention is potentially desirable from the perspective of the individuals. ${ }^{7}$

\footnotetext{
${ }^{6}$ This formulation is equivalent to choosing protection level $\delta(t) \in[0,1]$ at cost $\delta(t) c$, which then yields the infection rate $(1-\delta(t)) \beta I(t)$.

${ }^{7}$ Mathematically, it is a sufficient condition for the individual decision makers to switch between exposure and prevention for interior levels of disease prevalence and thus makes the decision non-trivial.
} 


\section{Assumption 2}

$$
\alpha<\beta
$$

This assumption implies that the eradication steady state of the classical model is unstable and the relevant steady state is the endemic one. Note that if $\alpha>\beta$, then the infectious disease is eventually eradicated even if no-one engages in any preventive behavior at all. The only possible role for preventive measures would then be to speed up the inevitable eradication of the disease.

\section{Centralized Decision Making}

In this section, I analyze the centralized problem. In this setup, a benevolent utilitarian social planner seeks to maximize the sum of the individuals' expected, discounted lifetime utilities through the direct control of aggregate exposure $\varphi(t) \in[0,1]$. Aggregate exposure $\varphi(t)$ can alternatively be interpreted as individuals' average exposure level or as the fraction of the susceptible population that are fully exposed (with the remainder fully protected).

Formally, the planner's problem is to solve the following programme:

$$
\begin{gathered}
\max _{\varphi(t) \in[0,1]} \int_{0}^{\infty} e^{-\rho t}\left[I(t) \pi_{\mathcal{I}}+S(t)\left(\pi_{\mathcal{S}}-(1-\varphi(t)) c\right)\right] d t \\
\text { s.t. } \quad \dot{I}(t)=I(t)[\varphi(t) \beta S(t)-\alpha], \quad I(0)=I_{0} \\
\dot{S}(t)=I(t)[\alpha-\varphi(t) \beta S(t)], \quad S(0)=1-I_{0}
\end{gathered}
$$

The constraints on the planner's problem are the laws of motion for the measures of infected and susceptible individuals, suitably modified to take into account that the rate at which infection occurs is a function of the centrally chosen aggregate exposure level.

Instead of solving this problem, the following simplified but equivalent programme will be considered (which differs only by the constant $\pi_{\mathcal{S}}$ in the objective and by using the normalization of the population size):

$$
\begin{aligned}
& \max _{\varphi(t) \in[0,1]} \int_{0}^{\infty} e^{-\rho t}[-I(t) \pi-(1-I(t))(1-\varphi(t)) c] d t \\
& \text { s.t. } \dot{I}(t)=I(t)[\varphi(t) \beta(1-I(t))-\alpha], \quad I(0)=I_{0}
\end{aligned}
$$

An admissible policy is a pair of functions $(I(t), \varphi(t))$ in which for all $t \geq 0, I(t)$ satisfies the logistic growth equation $(12)$ and $\varphi(t) \in[0,1]$. Furthermore, $\varphi(t)$ must be piecewise continuous. ${ }^{8}$ Let $\lambda(t)$ denote the costate variable, i.e. the shadow price of infection.

The following results characterize the optimal solution under centralized decision making:

Theorem 1. (i) The centralized problem has a unique steady state $\left(I_{C}^{*}, \lambda_{C}^{*}\right)$ and at this steady state, disease prevalence is interior. The steady state is given by

\footnotetext{
${ }^{8}$ The restriction to piece-wise continuous controls is without loss of optimality, since an optimal control exists within this class.
} 


$$
\begin{aligned}
I_{C}^{*} & \equiv \frac{c \rho}{\beta(\pi-c)} \\
\lambda_{C}^{*} & \equiv \frac{-(\pi-c)}{\rho}
\end{aligned}
$$

(ii) An optimal path exists and is of the most rapid approach type. It is characterized by

$$
\begin{aligned}
\varphi_{C}^{*} & =0 \text { for } I(t)>I_{C}^{*} \\
\varphi_{C}^{*} & =\frac{\alpha(\pi-c)}{\beta \pi-(\beta+\rho) c} \text { for } I(t)=I_{C}^{*} \\
\varphi_{C}^{*} & =1 \text { for } I(t)<I_{C}^{*}
\end{aligned}
$$

Proof: Part (i) follows from the steady state conditions $\dot{I}(t)=\dot{\lambda}_{C}(t)=0$, the planner's Hamiltonian condition and Assumption 1, which imply that there is a unique singular control and that this is interior and leads to an interior steady state. In the online Appendix, it is also proved that any steady state with a non-singular control does not satisfy the relevant transversality condition. The proof of part (ii) is in Appendix A

These results have a straightforward intuition. When enough individuals are infected, the probability that an unprotected individual will become infected is so high that the marginal cost of prevention is outweighed by the marginal expected welfare loss of additional infection. Similarly, when only a few individuals are infected, the probability of becoming infected is too low to warrant engaging in costly preventive behavior since the marginal cost of doing so is higher than the marginal social benefits. Thus the optimal policy always forces disease prevalence towards the interior of its domain.

Because of the bang-bang nature of the optimal policy, this means that whenever disease prevalence is above the steady state level, the planner optimally reduces exposure to zero until the steady state level is reached. At this point, the planner switches to partial exposure so as to maintain the steady state level of disease prevalence. Similarly, whenever disease prevalence is below the steady state level, the planner optimally chooses full exposure until disease prevalence has increased to its steady state level, which is subsequently maintained through partial exposure.

For later comparison, note that the switch in optimal exposure is determined by necessary Hamiltonian condition for optimality,

$$
\lambda(t) \beta I(t)+c=0
$$

This condition equalizes the social marginal benefits of exposure with its social marginal costs.

It is important to emphasize that while the optimal exposure policy is of the bangbang type, the main takeaways from the analysis do not depend on this feature. The important feature of the optimal policy to be noted here is that exposure is monotone decreasing in the risk faced by the decision maker, which generalizes straightforwardly to non-linear cost structures. ${ }^{9}$

\footnotetext{
${ }^{9}$ The fact that linearity is not restrictive in this class of models is also found by Goldman and Lightwood (2002) in a model of treatment. The extension to non-linear convex costs is discussed further in the online Appendix.
} 


\section{Decentralized Decision Making}

In this section, I analyze the decentralized problem. I first analyze the problem of an individual and then aggregate across the entire population.

From the perspective of an individual, the path of disease prevalence is exogenously given. ${ }^{10}$ It will be assumed that each individual has perfect foresight, in the sense that conjectures about the aggregate evolution of the disease are confirmed in equilibrium.

Let $q_{i}(t) \in[0,1]$ denote the probability that a randomly chosen individual in the population is infected at time $t \geq 0$. The individual's problem can then be written as follows: ${ }^{11}$

$$
\begin{gathered}
\max _{\varepsilon_{i}(t) \in[0,1]} \int_{0}^{\infty} e^{-\rho t}\left[-q_{i}(t) \pi-\left(1-q_{i}(t)\right)\left(1-\varepsilon_{i}(t)\right) c\right] d t \\
\text { s.t. } \quad \dot{q}_{i}(t)=\varepsilon_{i}(t) \beta I(t)\left(1-q_{i}(t)\right)-\alpha q_{i}(t), \quad q_{i}(0) \in\{0,1\}
\end{gathered}
$$

For an infected individual at instant $t \geq 0, q_{i}(t)=1$ and thus there is no decision to be made. For a susceptible individual at instant $t \geq 0, q_{i}(t)=0$ and hence the individual trades off costs and benefits of controlling the rate of transition from $\mathcal{S}(t)$ to $\mathcal{I}(t)$.

An admissible policy is a pair of functions $\left(q_{i}(t), \varepsilon_{i}(t)\right)$ in which for all $t \geq 0, q_{i}(t)$ satisfies the law of motion in (18) and where $\varepsilon_{i}(t) \in[0,1]$. Furthermore, $\varepsilon_{i}(t)$ must be piecewise continuous. ${ }^{12}$ Let $\mu(t)$ denote the costate variable, i.e. shadow price of the probability of being in the infected state.

The above problem is solved for each individual on the background of the aggregate evolution of the infectious disease, which is, described by the following modified logistic growth equation:

$$
\dot{I}(t)=I(t)[\varepsilon(t) \beta(1-I(t))-\alpha], \quad \varepsilon(t) \equiv \int_{i \in \mathcal{S}(t)} S(t)^{-1} \varepsilon_{i}(t) d i
$$

In this equation, $\varepsilon(t)$ denotes the aggregate level of exposure resulting from the susceptible individuals' disaggregate exposure levels. Because the aggregate exposure is normalized by the measure of susceptible individuals $S(t), \varepsilon(t)$ can be interpreted as a fraction. Note that in contrast to the case of central planning, aggregate infection $I(t)$ is not directly chosen, but is rather the outcome of decentralized decisions by individuals.

The following result characterizes equilibrium under decentralized decision making:

Theorem 2. (i) The decentralized problem has a unique steady state $\left(I_{D}^{*}, \mu_{D}^{*}\right)$ and at this steady state, disease prevalence is interior. The steady state is given by

$$
\begin{aligned}
I_{D}^{*} & \equiv \frac{c(\alpha+\rho)}{\beta(\pi-c)} \\
\mu_{D}^{*} & \equiv \frac{-(\pi-c)}{\alpha+\rho}
\end{aligned}
$$

\footnotetext{
${ }^{10}$ Because each agent is negligible and does not influence the aggregate, any feedback between an individual's action and other individuals' responses is ignored by the individual.

${ }^{11}$ For the detailed steps leading to this formulation, see the online Appendix.

${ }^{12}$ The restriction to piece-wise continuous controls is without loss of optimality as best response control exists within this class.
} 
(ii) In the unique decentralized equilibrium, for all times $t \geq 0$ and individuals $i \in$ $\mathcal{S}(t)$, equilibrium strategies are given by

$$
\begin{aligned}
\varepsilon_{i}^{*} & =0 \text { for } I(t)>I_{D}^{*} \\
\varepsilon_{i}^{*} & =\frac{\alpha(\pi-c)}{\beta \pi-c(\alpha+\beta+\rho)} \text { for } I(t)=I_{D}^{*} \\
\varepsilon_{i}^{*} & =1 \text { for } I(t)<I_{D}^{*}
\end{aligned}
$$

Proof: Part (i) follows from the steady state conditions $\dot{I}(t)=\dot{q}(t)=\dot{\mu}_{D}(t)=0$, the individual's Hamiltonian condition and Assumption 1, which show that there is a unique singular control for the individual's problem and that this is interior and leads to an interior steady state. In the online Appendix, it is also proved that any steady state with a non-singular control does not satisfy the relevant transversality condition. The proof of part (ii) is in Appendix A

Akin to the optimal policy in the centralized setup, in the decentralized equilibrium individuals play pure strategies outside of steady state in order to approach the singular solution as rapidly as possible. Once there, in the symmetric equilibrium the individuals switch to mixed strategies to maintain the steady state level of disease prevalence. The proof follows similar steps as those in the centralized setup.

The switch in privately optimal exposure is determined by necessary Hamiltonian condition for optimality:

$$
\mu(t) \beta I(t)+c=0
$$

This condition equalizes the private marginal benefits of exposure with its private marginal costs.

For reasons that parallel those in the centralized setup, the steady state is necessarily interior under decentralization. But because individuals do not internalize the effects that their preventive behavior has on other individuals and because each individual has a negligible effect on the aggregate, the centralized and aggregate decentralized steady state policies and disease prevalences differ. To better understand these differences, it is worthwhile briefly discussing the nature of externalities in the model.

First, along a path to steady state, all individuals either fully expose or fully protect themselves irrespective of what others do. Once in steady state, in a symmetric equilibrium all susceptible individuals mix between protection and exposure to maintain a constant level of disease prevalence. This means that away from steady state, there is no strategic interaction, while once at steady state, protection decisions are strategic substitutes. The former result stems from the linearity of costs which yields bang-bang policies. With more general convex cost structures, strategic substitutability would obtain also on transition paths and not only at steady state.

Second, as will be explored further below, individuals expose themselves too much from a social welfare perspective. The discrepancy between socially optimal and equilibrium exposure has two sources. On one hand, individuals do not internalize the fact that their exposure decisions influence the wellbeing of other individuals. This is a classic instance of an uninternalized negative externality, which causes individuals to overexpose themselves. On the other hand, the contemporaneous external effects just described has additional intertemporal effects. The reason is that individuals at each moment face an intertemporal tradeoff that weighs costs of protection against the benefits from exposure. 


\begin{tabular}{|c|c|c|c|c|c|c|}
\hline \multirow{2}{*}{} & \multicolumn{2}{|c|}{ Steady State Exposure } & \multicolumn{2}{c|}{ Steady State Prevalence } & \multicolumn{2}{c|}{ Steady State Welfare } \\
\cline { 2 - 7 } & Centralized & Decentralized & Centralized & Decentralized & Centralized & Decentralized \\
\hline \hline & $\frac{\alpha(\pi-c)}{\beta \pi-(\beta+\rho) c}$ & $\frac{\alpha(\pi-c)}{\beta \pi-(\alpha+\beta+\rho) c}$ & $\frac{c \rho}{\beta(\pi-c)}$ & $\frac{c(\alpha+\rho)}{\beta(\pi-c)}$ & $\frac{-c(\beta+\rho-\alpha)}{\beta}$ & $\frac{-c(\beta+\rho)}{\beta}$ \\
\hline$\alpha$ & + & + & 0 & + & + & 0 \\
\hline$\beta$ & - & - & - & - & + for $\rho>\alpha$ & + \\
\hline$\pi$ & - & - & - & - & 0 & 0 \\
\hline$c$ & + & + & + & + & - & - \\
\hline$\rho$ & + & + & + & + & - & - \\
\hline
\end{tabular}

Table 1: Steady state values and comparative statics results. A $(+)$ indicates that the steady state value is increasing in the variable in the first column, while a ( - ) indicates that it is decreasing.

This tradeoff is a function of the level of disease prevalence in the population, to wit on the entire future path of infection. Since exposure decisions under decentralized decision making are suboptimally high, the future equilibrium path of infection is strictly higher than that induced under centralized decision making. This means that from the perspective of an individual, the future path of infection is different from that facing the central planner and this discrepancy makes their tradeoffs different, even controlling for the contemporaneous external effects. Higher future disease prevalence means that the individuals will find it optimal to engage in lower future exposure, somewhat offsetting the direct contemporaneous external effect. The intertemporal effects of the externality are due to the fact that each individual is numerically insignificant and therefore takes the future path of infection as given, in contrast to the central planner who directly controls this path. For further analysis of these issues, including a formal decomposition of such effects, see the online Appendix and Rowthorn and Toxvaerd (2015).

\section{Welfare, Policy and Rational Disinhibition}

In this section, I consider two specific second-best policy measures, namely medical interventions that (i) permanently alter the infectiousness of the disease and (ii) permanently alter the rate of recovery from infection.

For ease of reference, the steady state values and the comparative statics results for the centralized and decentralized settings are summarized in Table 1.

Direct inspection shows that the steady state value of prevalence under centralized decision making is interior. It follows that in this model, it is never optimal to eradicate the disease through moment-by-moment protection. This should be contrasted to the possible desirability of eradicating the disease though other means, such as vaccination or treatment. ${ }^{13}$

Second, direct comparison of the steady state values confirms that when $\alpha>0$, individuals value protection less than the social planner does. Since prevention is valued less by individuals than by the social planner, the former will tend to choose higher exposure than the level preferred by the latter, which will in turn lead to higher disease

\footnotetext{
${ }^{13}$ Rowthorn and Toxvaerd (2015) show that a policy that involves eradication does not satisfy the relevant transversality condition. Furthermore, they show that with sufficiently ineffective prevention, there may be steady states in which full prevention is optimal. But under such scenarios, the disease is never eradicated.
} 
prevalence and to lower steady state flow welfare.

5.1. Interventions that Influence Infectiousness. Next, I will analyze the effects and desirability of permanently changing the infectiousness of the disease. Before doing so, there are a number of common (and somewhat overlapping) misconceptions that must be addressed.

The first mistake, which is now recognized by some in both the policy and the academic communities, is the failure to explicitly account for behavioral responses to changes in biomedical or economic parameters. A policy based on the classical epidemiological propagation equation, which ignores behavioral responses, would recommend a decrease in $\beta$ in order to decrease disease incidence. But the comparative statics of the classical model are inadequate for policy analysis as they take individuals' behavior as given and fixed. As is clear from the present analysis, any decision maker that trades off costs and benefits of exposure will respond to changes in infectivity $\beta$. Thus the mechanistic disease accounting inherent in the classical approach is inappropriate for policy analysis. In short, while decreasing infectivity $\beta$ indeed reduces overall disease incidence, given exposure levels, these in turn increase in response to the decrease in infectivity and thus cannot be taken as given.

The second mistake, which is still ubiquitous in the policy debate on pre-exposure prophylaxis, is to confound statements about the presence of behavioral disinhibition with statements about the desirability of interventions that reduce risk. That is, it is common to see judgements about social welfare based solely on the presence (or lack of) disinhibition. ${ }^{14}$ As shown here, such a conclusion is unwarranted, simply because both the central planner and the individuals respond to decreased infectivity by increasing the exposure to infection. Thus the presence (or lack of) disinhibition is not an appropriate yardstick with which to measure changes in social welfare.

Rather than lumping the response of the social planner and that of the individuals into the common category "behavioral disinhibition" as is commonly done, it is useful to distinguish between optimal disinhibition (exhibited by the central planner) and rational disinhibition (exhibited by individuals). The latter is rational in the sense that it is consistent with individual discounted expected utility maximization. With these concepts in place, it follows that behavioral disinhibition should in general be expected, but that it need not in itself be a cause for concern. ${ }^{15}$

The third common mistake is the failure to identify the source of potential gains from permanently reducing infectivity. In the present model, the welfare gains flowing from a permanent decrease in infectivity stem from the benefits of increased exposure, rather than from permanently lower steady state disease prevalence. In other words, if a decrease in infectivity increases overall welfare, it is because on the path towards the new and higher steady state, individuals fully expose themselves to infection. It is precisely the welfare gains associated with the higher exposure levels on the transition path that account for the overall increase in welfare. In short, welfare is increased despite the fact that it leads to higher levels of infection.

\footnotetext{
${ }^{14}$ See e.g. Abbas et al. (2007), Over (2008), Over et al. (2004) and Szekeres et al. (2004).

${ }^{15}$ Risk compensation is documented by Cohen et al. (2009), Paltiel et al. (2009) and Crepaz et al. (2004). See also discussions in Philipson and Posner (1993), Szekeres et al. (2004) and Blower et al. (2000).
} 
Next, I formalize the above observations by considering the following thought experiment. Suppose as a benchmark that the initial infectivity parameter is given by a baseline value $\beta_{1}$, which leads to an associated steady state prevalence level $I_{1} \equiv I_{D}^{*}\left(\beta_{1}\right)$ under decentralized decision making. Assume that the system has reached this steady state. Next, consider an exogenous, costless and permanent reduction of infectiousness to some lower rate $\beta_{2}<\beta_{1}$ and denote by $I_{2} \equiv I_{D}^{*}\left(\beta_{2}\right)$ the associated steady state prevalence level.

To evaluate the welfare consequences of reducing infectivity $\beta$ in this way, I compare the discounted social values of (i) staying indefinitely at steady state $I_{1}$ under infectivity $\beta_{1}$ and (ii) taking the path that moves the system from the old steady state $I_{1}$ to the new steady state $I_{2}$ and stays there indefinitely, the latter path calculated under infectivity $\beta_{2}$.

It follows from the comparative statics results in Table 1 that $I_{2}>I_{1}$ and the associated steady state welfare levels are such that $W_{D}\left(\beta_{2}\right)<W_{D}\left(\beta_{1}\right)$. In other words, under decentralized decision making, a reduction in infectivity increases both equilibrium steady state exposure and disease prevalence, leading to a decrease in steady state social welfare. ${ }^{16}$ If the discounted social welfare associated with option (i) is higher than the discounted social welfare of option (ii), then reducing infectivity is not a welfare enhancing policy and there is immiserization.

Let $V\left(I, I^{\prime} ; \beta\right)$ denote the discounted social value of following the most rapid approach path that starts at steady state $I$ and ends (and stays perpetually) at steady state $I^{\prime}$, under infectivity $\beta$. With this notation, there is immiserization of the population if for some values $\left(\beta_{1}, \beta_{2}\right)$ with $\beta_{2}<\beta_{1}$ it is the case that

$$
V\left(I_{1}, I_{2} ; \beta_{2}\right)<V\left(I_{1}, I_{1} ; \beta_{1}\right)
$$

where the latter term is simply the discounted social welfare of staying indefinitely at steady state $I_{1}$.

Turning to the social planner, let $I_{1}^{*} \equiv I_{C}^{*}\left(\beta_{1}\right)$ and $I_{2}^{*} \equiv I_{C}^{*}\left(\beta_{2}\right)$ denote the end points of the optimal paths under centralized decision making under the two infectivity levels being considered. Because the planner can directly control the path of aggregate disease prevalence, it can ensure itself at least the value $V\left(I_{1}^{*}, I_{1}^{*} ; \beta_{2}\right)$, i.e. the value of staying forever at the original steady state, but calculated using the lower infectivity rate $\beta_{2}$. By re-optimizing over possible paths, it will typically do even better. In other words, the following result holds:

Theorem 3. Under centralized decision making,

$$
V\left(I_{1}^{*}, I_{2}^{*} ; \beta_{2}\right) \geq V\left(I_{1}^{*}, I_{1}^{*} ; \beta_{2}\right)>V\left(I_{1}^{*}, I_{1}^{*} ; \beta_{1}\right)
$$

In summary, a planner that permanently decreases infectivity and optimally steers disease prevalence from some steady state $I_{1}^{*}$ to $I_{2}^{*}$ necessarily increases overall discounted social welfare. This is in contrast to the situation under decentralized decision making,

\footnotetext{
${ }^{16}$ Gersovitz (2010) states that "A sufficient condition for an improvement in welfare caused by a change that is a technological improvement (one that increases welfare in the social planner's problem) is [..] that the rate of infection fall in the long-run steady state". As seen above, this condition is never satisfied in the present model and thus has no bite, i.e. immiserization cannot be ruled out.
} 


\begin{tabular}{|l|c|l|c|c|}
\hline & Status Quo & Intervention & Status Quo & Intervention \\
\hline \hline Dec. & $I_{1}=0.001871$ & $I_{2}=0.002041$ & $V\left(I_{1}, I_{1} ; \beta_{1}\right)=-0.916667$ & $V\left(I_{1}, I_{2} ; \beta_{2}\right)=-0.917353$ \\
\hline Cen. & $I_{1}^{*}=0.000187$ & $I_{2}^{*}=0.000204$ & $V\left(I_{1}^{*}, I_{1}^{*}, \beta_{1}\right)=-0.766667$ & $V\left(I_{1}^{*}, I_{2}^{*} ; \beta_{2}\right)=-0.754725$ \\
\hline
\end{tabular}

Table 2: Example of immiserization.

where the reduction in infectivity causes a shift from steady state $I_{1}$ to $I_{2}$. From a social perspective, neither steady state $I_{1}$ nor $I_{2}$ is the end point of an optimal path. Thus the reduction in infectivity involves moving from one socially suboptimal steady state to another socially suboptimal steady state. There is therefore no reason to expect such a transition to be welfare enhancing in general.

For a concrete example of how welfare losses can result from a permanent lowering of the infectiousness of the disease, consider a reduction from $\beta_{1}=6$ to $\beta_{2}=5.5$ with the other parameters given by $\alpha=1, \rho=0.11, c=0.1$ and $\pi=10$. Under centralized decision making, this reduction of infectiousness takes the system from steady state prevalence $I_{1}^{*}=0.000187$ to $I_{2}^{*}=0.000204$, in the process increasing discounted social welfare from $V\left(I_{1}^{*}, I_{1}^{*}, \beta_{1}\right)=-0.766667$ to $V\left(I_{1}^{*}, I_{2}^{*} ; \beta_{2}\right)=-0.754725$. Turning to decentralized decision making, the same reduction in infectiousness takes the system from steady state disease prevalence $I_{1}=0.001871$ to $I_{2}=0.002041$. In contrast to the the situation under central planning, under decentralized decision making this reduction in infectiousness decreases discounted social welfare, from $V\left(I_{1}, I_{1} ; \beta_{1}\right)=-0.916667$ to $V\left(I_{1}, I_{2} ; \beta_{2}\right)=$ -0.917353 . For ease of comparison, I summarize these numbers in Table 2.

Although $W_{D}\left(\beta_{1}\right)>W_{D}\left(\beta_{2}\right)$, so the decrease in infectiousness decreases steady state welfare under decentralized decision making, $W_{C}\left(\beta_{1}\right)<W_{C}\left(\beta_{2}\right)$ for this parameterization (since $\alpha=1>0.11=\rho$ ). This means that under centralized decision making, there is no tradeoff between gains on the transition path and losses from being in a new steady state; both contribute towards increased social welfare. Under decentralized decision making though, the new steady state is indeed worse and in this case the decrease in discounted steady state welfare dominates the benefits of increased exposure on the transition path.

For completeness, it should be emphasized that the criterion used to detect welfare improvements used in this thought experiment is very strict, because it assumes that infectivity can be reduced at no cost. Clearly, introducing the more realistic assumption that reducing infectivity is in fact costly would only further reduce the desirability of the policy in question.

It follows from this example that although reducing the infectiousness of a disease permanently has strong intuitive appeal, a more careful analysis is warranted. Such an analysis shows that the policy may lead to immiserization and therefore be counterproductive and negate the intended policy aim.

In the online Appendix, I outline results from more extensive numerical analysis. The main points of this analysis are that (i) immiserization can indeed occur for a wide set of parameter values, (ii) immiserization typically emerges for moderate decreases in infectiousness but not for very significant decreases and (iii) the presence and severity of immiserization depends on all the parameters of the model but the dependence is fairly complicated as the entire paths depend on these. Baseline infectiousness and the recovery rate tend to be particularly influential in determining the presence and severity of immiserization. 
5.2. Interventions that Influence Recovery. Next, I turn to the effects of permanently increasing the recovery rate $\alpha$. It can be verified from direct inspection of the steady state values that for the special case $\alpha=0$, the equilibrium outcome under decentralized decision making coincides with that chosen by the central planner. The reason that the decentralized equilibrium is socially optimal when $\alpha=0$ is that that in a symmetric setting with bang-bang policies, all individuals switch from full exposure to full (and perfect) protection at the same threshold level of disease prevalence. But this means that when individuals choose to protect themselves, so do all others and there are thus no susceptible individuals left in the population that can suffer from the negative externalities. ${ }^{17}$

From the results reported in Table 1, one readily concludes that increasing the recovery rate must increase social welfare and cannot result in immiserization. ${ }^{18}$ To see this, note that under both centralized and decentralized decision making, an increase in the recovery rate $\alpha$ leads to an unambiguous increase in steady state exposure and on the path to the new steady state, there is full exposure. So on this count, the change in $\alpha$ increases welfare. In addition, steady state welfare is itself non-decreasing in the recovery rate $\alpha$ (and in the case of centralized decision making, strictly increasing). Thus there is no welfare tradeoff from such an increase in the recovery rate and it therefore cannot lead to immiserization.

Another issue that should be noted is that in practice, increasing the recovery rate is likely achieved by increasing either the quality or the extent of treatment of infected individuals (that is, if treatment is feasible). But if treatment is feasible and chosen optimally, then the analysis in the present paper no longer applies and more complicated policies must be considered. Once prevention and treatment are chosen in conjunction, the dynamics of the model become very complicated and there may be multiple steady states across which the comparative statics results vary (see Rowthorn and Toxvaerd, 2015 for details). Furthermore, if treatment is available and optimally chosen, then the optimal level of prevention may no longer correspond to the level derived here. Still, increasing $\alpha$ can be interpreted as an innovation in therapeutic interventions in a world in which a policy of complete and permanent treatment of infected individuals is in place for unmodeled reasons.

\section{Extensions to a Heterogeneous Population}

The analysis above was conducted under the assumption of homogeneity, both in terms of payoffs and in terms of susceptibility to infection. The first type of homogeneity is that all individuals face identical costs of protection and infection. The second type of homogeneity is concerned with the contact pattern and, implicitly, the degree of susceptibility to infection. For both types of homogeneity, it is of some interest to consider what might be learned by relaxing the maintained assumptions. ${ }^{19}$

First, assume that rather than individuals having identical costs of protection, their costs are drawn from a distribution $F$ on some interval $[\underline{c}, \bar{c}]$. Note that in this setting,

\footnotetext{
${ }^{17}$ These issues are discussed further in the online Appendix and in Chen and Toxvaerd (2014).

${ }^{18}$ So while decreasing $\alpha$ would indeed narrow the gap between social welfare under centralized and decentralized decision making, the level of the latter would also be decreased, making such a policy undesirable.

${ }^{19}$ Preciado et al. (2014) consider both types of heterogeneity, but their analysis is orthogonal to the present work as they do not consider social welfare or utility maximization.
} 
the best responses of individuals are analogous to those in the homogeneous setting, but with each agent having a different tolerance to infection risk. For example, for an individual $i \in \mathcal{S}(t)$, the infection level that determines the switch from full exposure to full protection will be a function of the idiosyncratic cost parameter $c_{i}$, given by the Hamiltonian condition

$$
\mu(t) \beta I(t)+c_{i}=0
$$

For a given set of parameters characterizing the individual and a given level of aggregate disease prevalence, this condition pins down individual behavior.

Next, I will describe the features of the steady state heuristically. Because each cost level $c_{i}$ implicitly defines a critical threshold of aggregate infection $I_{i}(t)$ that causes the individual to switch between exposure and non-exposure. Then for a given level of disease prevalence $I(t)$ one can determine which individuals choose not to expose themselves at that level. Consider a steady state $I^{*}$ in this setting. Because the willingness of individual $i \in \mathcal{S}(t)$ to expose itself is increasing in its $\operatorname{cost} c_{i}$, the steady state $I^{*}$ of aggregate disease prevalence will have an associated threshold cost $c^{*} \in[\underline{c}, \bar{c}]$ such that all individuals with $\operatorname{costs} c<c^{*}$ choose no exposure in steady state. The situation facing the remaining individuals, who all have costs $c \geq c^{*}$, resembles that under homogeneous costs. In particular, in steady state, all such high cost individuals will mix between full protection and full exposure, but will do so with different mixing probabilities. Since an individual must be indifferent between its strategies in order to be willing to mix and they all face the same infection risk, it must be the case that high cost individuals expose themselves with higher probability. Thus in such a steady state, the proportion $F\left(c^{*}\right)$ of lowest cost individuals choose full protection while the remainder $\left(1-F\left(c^{*}\right)\right)$ choose to randomize over their exposure decisions. In conclusion, in such a steady state $\left(I^{*}, c^{*}\right)$, a fraction of the population protect themselves at all times and are never infected, while the remainder of the population never protect themselves fully and cycle perpetually between recovery and infection. ${ }^{20}$ Now consider an exogenous decrease in the infectiousness parameter $\beta$. Such a change will shift the location of the cutoff individual $c^{*}$ and of steady state prevalence $I^{*}$ upwards and prompt both an increase in set of individuals that expose themselves and to an increase in the levels of exposure by each individual.

For the second type of heterogeneity, consider a model in which individuals differ in terms of infection risk, such as in the work of Pastor-Satorras and Vespignani (2001), Jackson and Rogers (2007) and Lopez-Pintado (2008). Specifically, assume that an individual $i \in \mathcal{S}(t)$ is characterized by its in-degree (or connectivity) $d_{i}>0$, interpreted as the number of contacts that the individual has per instant. Assume that individuals' degrees are exogenously given and characterized by the population-wide distribution $P(d)$. Denoting by $\rho_{d}(t)$ the probability that an individual with degree $d$ is infected, a randomly chosen individual from the population will be infected with probability

$$
\theta(t) \equiv \frac{\sum_{d} P(d) \rho_{d}(t) d}{E_{P}[d]}
$$

The probability $\theta(t)$ can be thought of as the per-degree infection probability for a sus-

\footnotetext{
${ }^{20}$ Note that in a setting with increasing convex costs of abstinence, a similar feature will be present. Higher cost individuals will expose themselves more and consequently constitute a higher proportion of the class of infected individuals (relative to their weight in the population).
} 
ceptible individual. Using a mean-field approximation, the Hamiltonian condition for an individual of degree $d_{i}$ is then

$$
\mu(t) \beta \theta(t) d_{i}+c=0
$$

It follows that the product $\theta(t) d_{i}$ plays the exact same role as aggregate prevalence $I(t)$ plays in the case of homogeneous mixing. ${ }^{21}$ From the perspective of the individual, the degree-weighted infection risk per degree $\theta(t)$ is exogenous and the same across individuals. The best responses of individuals, in this heterogeneous susceptibility/connectivity setting has the same character as that described in the main analysis of this paper. That is, the propensity to expose oneself is decreasing in the incoming risk of infection as captured by the individual's degree. To be specific, higher degree individuals face higher risks and will therefore counteract this higher infection risk by curtailing their exposure more.

Heuristically, a steady state in this setting consists of a pair $\left(\theta^{*}, d^{*}\right)$ such that all individuals with degrees larger than $d^{*}$ will fully protect themselves. Individuals with degrees smaller than $d^{*}$ will choose to mix between full exposure and full prevention, with higher degree individuals choosing exposure with lower probability. The steady state per-degree risk $\theta^{*}$ will then be the degree-weighted steady state infection probabilities of the individuals in the population, i.e. $\rho_{d}^{*}$. Again, an exogenous decrease in the infectivity parameter $\beta$ will cause an upward shift in exposure levels and the resulting per-degree risk $\theta^{*}$, as well as in disease prevalence.

In summary, both types of heterogeneity give rise to best responses that are qualitatively similar to those in the homogeneous setting. Having said that, equilibrium outcomes and steady states are considerably more involved in these cases. The nature of externalities under heterogeneity may be more complicated than under homogeneity and thus may influence both equilibrium outcomes and policy analysis.

\section{Discussion and Policy Conclusions}

In this paper, I analyze a simple model of disease propagation in which individuals may engage in privately costly preventive behavior (but cannot control their rate of recovery). I analyze the outcomes under both centralized decision making by a benevolent social planner and under decentralized decision making by non-cooperative, forward-looking individuals. I find that in the decentralized equilibrium, individuals over-expose themselves to infection compared to the socially optimal level and that this leads to socially suboptimal steady state disease prevalence and welfare levels. This is a result of the fact that individuals do not internalize the external effects of their private prevention decisions and because each have negligible effects on aggregate disease dynamics.

Next, I consider the desirability of different second-best policy measures aimed at reducing the wedge between private and public incentives to engage in prevention, such as permanently reducing the infectiousness of the disease through pre-exposure prophylaxis. I show that such a policy intervention may actually reduce overall discounted social welfare. This stems from the presence of behavioral disinhibition, which means that decision makers respond to lower infectiousness by increasing their exposure to infection. When considering such policies, the policy maker would therefore do well to keep in mind the maxim "First, do no harm". Encapsulating a central concept in medical ethics, the

\footnotetext{
${ }^{21}$ Note that the assumption of homogeneous mixing in the homogeneous setting amounts to a meanfield approximation.
} 
advice reminds the physician that interventions have both costs and benefits and that inactivity may be preferable to prescribing a course of action that does more harm than good. In this work, I explicitly show that for some parameter ranges, immiserization may well be the consequence of permanently reducing the disease's infectiousness. This calls for careful analysis before the introduction of such second-best policy interventions.

There are a number of policy conclusions that emerge from this analysis. The first conclusion is that a seemingly sensible policy such as decreasing the infectivity of a disease can lead to immiserization, i.e. to a decrease in overall welfare. This stems from a combination of (i) disinhibition and (ii) external effects due to self-interested behavior. This means in particular that before introducing such policies, policy makers would be well-advised to carefully consider the overall effects that may be induced and not simply rely on intuition.

The second conclusion is that when a policy such as pre-exposure prophylaxis is indeed welfare enhancing, it is precisely because it allows individuals to benefit more from the exposure that they find desirable. These benefits will counterweight the subsequent decrease in steady state welfare that results from the intervention. In fact, with an overly paternalistic approach to policy making that ignores the benefits of exposure, there would never be any grounds for decreasing infectivity in the first place, even if all individuals were to perfectly conform to the behavior mandated by the social planner.

Last, it should be emphasized that the policies considered in this paper are secondbest in nature. It is possible to achieve socially better outcomes by the introduction of carefully tailored fines and subsidies, as shown in Rowthorn and Toxvaerd (2015). Having said that, first-best policies of this kind are typically very complicated objects and may thus be non-trivial to implement.

APPENDIX

A. Proofs that Optimal Policies Exist and are of the Mrap Type

A.1. The Centralized Setting. This appendix confirms that the conditions of Sethi (1977), Theorem 3.1 (i)-(vi) are satisfied for the centralized and decentralized problems respectively. I start with the former of these. From the logistic growth equation, solve for the exposure rate to get the expression

$$
\varphi(t)=\frac{\dot{I}(t)+\alpha I(t)}{\beta I(t)(1-I(t))}
$$


Substituting this into the objective function and rearranging, yields the modified objective function

$\int_{0}^{\infty} e^{-\rho t}\left[-I(t)[\pi-c]-c\left(\frac{\beta-\alpha}{\beta}\right)+\left(\frac{c}{\beta I(t)}\right) \dot{I}(t)\right] d t=\int_{0}^{\infty} e^{-\rho t}[M(I(t))+N(I(t)) \dot{I}(t)] d t$

where

$$
\begin{aligned}
M(I(t)) & \equiv-c\left(\frac{\beta-\alpha}{\beta}\right)-I(t)(\pi-c) \\
N(I(t)) & \equiv \frac{c}{\beta I(t)}
\end{aligned}
$$

Next, define

$$
\begin{aligned}
\Delta^{C}(I(t)) & \equiv-\left(\rho N(I(t))+M^{\prime}(I(t))\right) \\
& =\frac{-\rho c}{\beta I(t)}+(\pi-c)
\end{aligned}
$$

First, note that $I(t)=I_{C}^{*}$ is the unique solution to the equation

$$
\Delta^{C}(I(t))=0
$$

Next, given $I(t)=I_{C}^{*}, \varphi(t)=\varphi_{C}^{*}$ is the unique solution to the equation

$$
\dot{I}(t)=0
$$

Last, it is easy to verify that $\Delta^{C}(I(t))>0$ for $I(t)>I_{C}^{*}$ while $\Delta^{C}(I(t))<0$ for $I(t)<I_{C}^{*}$. This proves that conditions (i)-(iii) are satisfied. Conditions (iv)-(vi) hold trivially

A.2. The Decentralized Setting. Turning to the decentralized problem, solve the differential equation governing the probability of being infected for the individual exposure rate, to obtain

$$
\varepsilon(t)=\frac{\dot{q}_{i}(t)+\alpha q_{i}(t)}{\beta I(t)\left(1-q_{i}(t)\right)}
$$

Substitute into the individual's objective function and rearrange to get

$$
\int_{0}^{\infty} e^{-\rho t}\left[-q_{i}(t)\left(\pi-c-\frac{\alpha c}{\beta I(t)}\right)-c+\left(\frac{c}{\beta I(t)}\right) \dot{q}_{i}(t)\right] d t=\int_{0}^{\infty} e^{-\rho t}\left[\widehat{M}(I(t))+\widehat{N}(I(t)) \dot{q}_{i}(t)\right] d t
$$

where

$$
\begin{aligned}
\widehat{M}(I(t)) & \equiv-q_{i}(t)\left(\pi-c-\frac{\alpha c}{\beta I(t)}\right)-c \\
\widehat{N}(I(t)) & \equiv \frac{c}{\beta I(t)}
\end{aligned}
$$


Next, define

$$
\begin{aligned}
\Delta^{D}(I(t)) & \equiv-\left(\rho \widehat{N}(I(t))+\widehat{M}^{\prime}(I(t))\right) \\
& =\frac{-(\rho+\alpha) c}{\beta I(t)}+(\pi-c)
\end{aligned}
$$

Here, I have made the substitution $q_{i}(t)=I(t)$. It is easily verified that $I(t)=I_{D}^{*}$ is the unique solution to the equation

$$
\Delta^{D}(I(t))=0
$$

Given $q_{i}(t)=I(t)=I_{D}^{*}, \varepsilon_{i}(t)=\varepsilon_{D}^{*}$ is the unique solution to the equation

$$
\dot{q}_{i}(t)=0
$$

The last step is to note that $\Delta^{D}(I(t))>0$ for $I(t)>I_{D}^{*}$ while $\Delta^{D}(I(t))<0$ for $I(t)<I_{D}^{*}$. Conditions (iv)-(vi) hold trivially. This completes the proof

\section{REFERENCES}

[1] Aadland, D., D. Finnoff And K. X. D. Huang (2013): Syphilis Cycles, B.E. Journal of Economic Analysis $\&$ Policy, 13(1), 297-348.

[2] Abbas, U. L., R. M. Anderson and J. W. Mellors (2007): Potential Impact of Antiretroviral Chemoprophylaxis on HIV-1 Transmission in Resource-Limited Settings, PLoS ONE, 2(9): e875.

[3] Auld, M. C. (2003): Choices, Beliefs, and Infectious Disease Dynamics, Journal of Health Economics, 22(3), 361-377.

[4] Barrett, S. (2003): Global Disease Eradication, Journal of the European Economic Association, 1(2-3), 591-600.

[5] Bate, A. M., G. Jones, A. Kleczkowski, A. Macleod, R. Naylor, J. TimMis, J. M. TouzA And P. C. L. White (2016): Modelling the Impact and Control of an Infectious Disease in a Plant Nursery with Infected Plant Material Inputs, Ecological Modelling, 24(334), 27-43.

[6] Blower, S. M., H. B. Gershengorn and R. M. Grant (2000): A Tale of Two Futures: HIV and Antiretroviral Therapy in San Francisco, Science, 287(5453), 650-654.

[7] Blumenthal, J. And R. H. Haubrich (2014): Will Risk Compensation Accompany Pre-Exposure Prophylaxis for HIV?, AMA Journal of Ethics, 16(11), 909-915.

[8] Cerdeiro, D. (2017): Contagion Exposure and Protection Technology, Games and Economic Behavior, 105, 230-254.

[9] Chan, N. W. And K. Gillingham (2015): The Microeconomic Theory of the Rebound Effect and its Welfare Implications, Journal of the Association of Environmental and Resource Economists, 2(1), 133-159. 
[10] Chan, T. Y., B. H. Hamilton and N. W. Papageorge (2018): The Value of Pharmaceutical Innovation: AIDS, Antivirals and the Joy of (Risky) Sex, mimeo.

[11] Chen, F. And F. Toxvaerd (2014): The Economics of Vaccination, Journal of Theoretical Biology, 363, 105-117.

[12] Cohen, C. R., M. Montandon, A. W. Carrico, S. Shiboski, A. Bostrom, A. Obure, Z. Kwena, R. C. Bailey, R. Nguti and E. A. Bukusi (2009): Association of Attitudes and Beliefs towards Antiretroviral Therapy with HIVSeroprevalence in the General Population of Kisumu, Kenya, PLoS ONE, 4(3): e4573.

[13] Crepaz, N., T. A. Hart and G. Marks (2004): Highly Active Antiretroviral Therapy and Sexual Risk Behavior: A Meta-Analytic Review, Journal of the American Medical Association, 292(2), 224-36.

[14] Duranton, G. And M. A. Turner (2009): The Fundamental Law of Road Congestion: Evidence from US Cities, NBER Working Paper No. 15376.

[15] Duranton, G. And M. A. Turner (2011): The Fundamental Law of Road Congestion: Evidence from US Cities, American Economic Review, 101(6), 2616-2652.

[16] Francis, P. J. (2004): Optimal Tax/Subsidy Combinations for the Flu Season, Journal of Economic Dynamics \&s Control, 28(10), 2037 - 2054.

[17] Geoffard, P.-Y. and T. Philipson (1996): Rational Epidemics and Their Public Control, International Economic Review, 37(3), 603-624.

[18] Gersovitz, M. (2010): Disinhibition and Immiserization in a Model of SusceptibleInfected-Susceptible (SIS) Diseases, mimeo.

[19] Gersovitz, M. And J. S. Hammer (2004): The Economical Control of Infectious Diseases, Economic Journal, 114(492), 1-27.

[20] Gillingham, K., D. Rapson and G. Wagner (2015): The Rebound Effect and Energy Efficiency Policy, Review of Environmental Economics and Policy, 10(1), 68-88.

[21] Goldman, S.M. And J. Lightwood (2002): Cost Optimization in the SIS Model of Infectious Disease with Treatment, Topics in Economic Analysis and Policy, 2(1), $1-22$.

[22] Greenwood, J., P. Kircher, C. Santos And M. Tertilt (2013): An Equilibrium Model of the Malawian HIV/AIDS Epidemic, mimeo.

[23] Hoy, M. And M. K. Polborn (2015): The Value of Technology Improvements in Games with Externalities: A Fresh Look at Offsetting Behavior, Journal of Public Economics, 131, 12-20.

[24] Jackson, M. O. And B. W. Rogers (2007): Relating Network Structure to Diffusion Properties through Stochastic Dominance, B.E. Journal of Theoretical Economics, 7(1), Article 6 . 
[25] Keeling, M. J. And P. Rohani (2008): Modeling Infectious Diseases in Humans and Animals, Princeton University Press.

[26] Kremer, M. (1996): Integrating Behavioral Choice into Epidemiological Models of AIDS, Quarterly Journal of Economics, 111(2), 549-573.

[27] Kremer, M. And C. M. Snyder (2003): Why Are Drugs More Profitable Than Vaccines?, NBER Working Paper, No. 9833.

[28] Lakdawalla, D., N. Sood And D. Goldman (2006): HIV Breakthroughs and Risky Sexual Behavior, Quarterly Journal of Economics, 121(3), 1063-1102.

[29] Lopez-Pintado, D. (2008): Diffusion in Complex Social Networks, Games and Economic Behavior, 62(2), 573-590.

[30] Over, M. (2008): Opportunities for Presidential Leadership on AIDS: From an "Emergency Plan" to a Sustainable Policy, in The White House and the World: A Global Development Agenda for the Next U.S. President, N. Birdsall (ed.), Center for Global Development, Washington, D.C.

[31] Over, M., P. Heywood, J. Gold, I. Gupta, S. Hira and E. Marseille (2004): HIV/AIDS Treatment and Prevention in India. Modeling the Costs and Consequences, The World Bank.

[32] Paltiel, A. D., K. A. Freedberg, C. A. Scott, B. R. Schackman, E. Losina, B. Wang, G. R. Seage III, C. E. Sloan, P. E. Sax, and R. P. Walensky (2009): HIV Preexposure Prophylaxis in the United States: Impact on Lifetime Infection Risk, Clinical Outcomes, and Cost-Effectiveness, Clinical Infectious Diseases, 48, 806-815.

[33] Pastor-Satorras, R. And A. Vespignani (2001): Epidemic Spreading in ScaleFree Networks, Physical Review Letters, 86(14), 3200-3203.

[34] Peltzman, S. (1975): The Effects of Automobile Safety Regulation, Journal of Political Economy, 83(4), 677-726.

[35] Philipson, T., and R. A. Posner (1993): Private Choices and Public Health: An Economic Interpretation of the AIDS Epidemic. Cambridge, MA: Harvard University Press.

[36] Preciado, V. M., M. Zargham, C. Enyioha, A. Jadbabaie and G. PapPAS (2014): Optimal Resource Allocation for Network Protection Against Spreading Processes, IEEE Transactions on Control of Network Systems, 1(1), 99-108.

[37] Reluga, T. C. (2009): An SIS Epidemiology Game with Two Subpopulations, Journal of Biological Dynamics, 1751-3766.

[38] Reluga, T. C. (2010): Game Theory of Social Distancing in Response to an Epidemic, PLoS Computational Biology, 6(5). 
[39] Richens, J., J. Imrie And A. Copas (2000): Condoms and Seat Belts: The Parallels and the Lessons, The Lancet, 355, 400-403.

[40] Rowthorn, R. And F. Toxvaerd (2015): The Optimal Control of Infectious Diseases via Prevention and Treatment, mimeo.

[41] Sethi, S. P. (1977): Nearest Feasible Paths in Optimal Control Problems: Theory, Examples, and Counterexamples, Journal of Optimization Theory and Applications, 23(4), 563-579.

[42] Sethi, S. P. (1978): Optimal Quarantine Programmes for Controlling an Epidemic Spread, Journal of the Operational Research Society, 29(3), 265-268.

[43] Szekeres, G., T. J. Coates, S. Frost, A. Leibowitz and S. Shoptaw (2004): Anticipating the Efficacy of HIV Pre-Exposure Prophylaxis (PrEP) and the Needs of At-Risk Californians, Center for HIV Identification, Prevention, and Treatment Services (CHIPTS).

[44] Talamas, E. And R. Vohra (2018): Go Big or Go Home: A Free and Perfectly Safe but Only Partially Effective Vaccine Can Make Everyone Worse Off, PIER Working Paper No. 18-006. 